\title{
Diagnósticos de enfermagem da taxonomia NANDA-I para idosos em instituição de longa permanência
}

\author{
Nursing diagnoses of NANDA-I taxonomy for the elderly in a long-term institution
}

Diagnósticos de enfermería de la taxonomía NANDA-I para ancianos en una institución a largo plazo

\author{
Edileuza Teixeira Santana ${ }^{1}$ (C) \\ Gabriella Gonçalves Coutinho ${ }^{1}$ (D) \\ Daniel Vinícius Alves Silva² (1) \\ Tatielle Aparecida Almeida Bernardes ${ }^{1}$ (1) \\ Luiza Rodrigues Camisasca ${ }^{1}$ (D) \\ Ricardo Otávio Maia Gusmão ${ }^{3}$ (1) \\ Diego Dias de Araújo $^{3}$ (C)
}

1. Universidade Estadual de Montes Claros.

Montes Claros, MG, Brasil.

2. Irmandade Nossa Senhora das Mercês de Montes Claros - Santa Casa. Montes Claros, MG, Brasil.

3. Universidade Estadual de Montes Claros, Departamento de Enfermagem. Montes

Claros, MG, Brasil.

\section{Resumo}

Objetivo: identificar diagnósticos de enfermagem da Taxonomia da NANDA-I em idosos institucionalizados. Método: estudo descritivo, conduzido com 116 idosos de uma instituição de longa permanência, realizado a partir da identificação de diagnósticos de enfermagem da Taxonomia da NANDA-I em um instrumento fundamentado no modelo teórico das Necessidades Humanas Básicas e validação por especialistas com suporte no raciocínio diagnóstico de Risner. Resultados: identificou-se 1.555 títulos de diagnósticos de enfermagem para os 116 idosos, com uma média de 13,4 diagnósticos por idoso. Após exclusão de repetições foram obtidos 39 títulos distintos de diagnósticos. Entre eles, 26 (66,7\%) são títulos de diagnósticos reais, $13(33,3 \%)$ títulos de diagnósticos de risco e estão classificados em 8 dos 13 domínios da taxonomia da NANDA-I. Os diagnósticos de enfermagem mais frequentes foram: risco de quedas (94,8\%); síndrome do idoso frágil $(91,3 \%)$ e processos familiares disfuncionais $(90,5 \%)$. Conclusão e implicações para a prática: o perfil de diagnósticos de enfermagem identificado pode contribuir para o incremento de indicadores sensiveis à prática de enfermagem, com o planejamento e implementação de medidas assistenciais direcionadas as reais necessidades dos idosos institucionalizados impactando na autonomia, independência $e$ consequentemente na qualidade de vida.

Palavras-chave: Idoso; Instituição de Longa Permanência para Idosos; Saúde do Idoso; Enfermagem; Diagnóstico de Enfermagem.

\section{Abstract}

Objective: to identify nursing diagnoses of NANDA-I Taxonomy in institutionalized elderly. Method: a descriptive study, conducted with 116 elderly people from a long-term institution, carried out based on the identification of nursing diagnoses of NANDA-I Taxonomy in an instrument based on the theoretical model of Basic Human Needs and validation by experts with support in reasoning Risner's diagnosis. Results: 1.555 nursing diagnosis titles were indicated for the 116 elderly people, with an average of 13.4 diagnoses per elderly person. After excluding repetitions, 39 different diagnostic titles were obtained. Among them, 26 (66.7\%) are titles of real diagnoses, $13(33.3 \%)$ are titles of risk diagnoses, and are classified in 8 of the 13 domains of the NANDAtaxonomy. The most frequent nursing diagnoses were: risk of falls (94.8\%); frail elderly syndrome (91.3\%) and dysfunctional family processes $(90.5 \%)$. Conclusion and implications for the practice: the profile of nursing diagnoses identified can contribute to the increase of sensitive indicators to nursing practice, with the planning and implementation of care measures directed to the real needs of institutionalized elderly, impacting on autonomy, independence and consequent on quality of life.

Keywords: Aged; Homes for the Aged; Health of the Elderly; Nursing; Nursing Diagnosis.

\section{REsumen}

Objetivo: identificar diagnósticos de enfermería de la taxonomía NANDA-I en ancianos institucionalizados. Método: estudio descriptivo, realizado con 116 personas mayores de una residencia gerontológica, a partir de la identificación de diagnósticos de enfermería de la taxonomía NANDA-I en un instrumento basado en el modelo teórico de Necesidades Humanas Básicas y validado por especialistas con apoyo en razonamiento Diagnóstico de Risner. Resultados: se indicaron 1.555 títulos de diagnóstico de enfermería para los $116 \mathrm{p}$ edad adultos mayores, con un promedio de 13.4 diagnósticos por adulto mayor. Excluidas las repeticiones, se obtuvieron 39 títulos de diagnóstico diferentes. Entre ellos, 26 (66.7\%) son títulos de diagnósticos reales, 13 (33.3\%) son títulos de diagnósticos de riesgo, y se clasifican en 8 de los 13 dominios de la taxonomía NANDA-I. Los diagnósticos de enfermería más frecuentes fueron: riesgo de caídas (94,8\%); síndrome de fragilidad del anciano (91.3\%) y procesos familiares disfuncionales $(90.5 \%)$. Conclusión e implicaciones para la práctica: el perfil de los diagnósticos de enfermería identificados puede contribuir al aumento de indicadores sensibles a la práctica de enfermería, con la planificación e implementación de medidas de atención dirigidas a las necesidades reales de los ancianos institucionalizados, que impactan en la autonomía, independencia y consecuentemente calidad de vida.

Palabras clave: Anciano; Hogares para Ancianos; Salud del Anciano; Enfermería; Diagnóstico de Enfermería.
Autor correspondente Diego Dias de Araújo diego.araujo@unimontes.br

\section{Recebido em 02/04/2020.}

Aprovado em 21/06/2020. 


\section{INTRODUÇÃO}

O envelhecimento populacional é uma conquista da humanidade e também um dos maiores desafios a ser enfrentado pela sociedade. No século XXI, o envelhecimento aumentará mundialmente as demandas de saúde, sociais e econômicas. ${ }^{1}$

A Organização das Nações Unidas (ONU), em 1985, estabeleceu a idade igual ou superior a 65 anos para classificar idosos em países desenvolvidos. Entretanto, nos países em desenvolvimento, onde a expectativa de vida é menor, como é o caso do Brasil, determinou-se a idade de 60 anos ou mais, e esse entendimento é reforçado pelo Ministério da Saúde do Brasil pela Política Nacional do Idoso. ${ }^{2}$

De acordo com estimativas estatísticas do Instituto Brasileiro de Geografia e Estatística (IBGE), a população brasileira está em trajetória de envelhecimento e até o ano de 2060, o percentual de pessoas com mais de 60 anos passará dos $9,2 \%$ da atualidade para $25,5 \%$. Ou seja, um em cada quatro brasileiros será idoso. ${ }^{3}$

Constata-se que o envelhecimento populacional ocasiona alterações socioeconômicas importantes, como o aumento da demanda e da necessidade de reorganização de serviços de saúde e de profissionais qualificados para atender os idosos. ${ }^{4}$ Apesar de ser um fenômeno natural, envelhecer impacta no estado de saúde, consequentemente, o idoso é um indivíduo mais susceptível à fragilidade. Dentre outras questões para além da saúde, o envelhecimento pode resultar na necessidade de referenciar o idoso a uma Instituição de Longa Permanência para Idosos (ILPI). ${ }^{5}$

Segundo a Agência Nacional de Vigilância Sanitária, ILPIs são instituições governamentais ou não-governamentais, de caráter residencial, destinadas a acolher na modalidade de domicílio coletivo, idosos com ou sem suporte familiar, em condição de liberdade, dignidade e cidadania. ${ }^{6,7}$

Conforme a Lei no $7.498 / 86^{8}$ e a Resolução do Conselho Federal de Enfermagem (COFEN), $n^{\circ} 620 / 19$, foi regulamentado o exercício da Enfermagem em todo o território nacional e normatizadas as atribuições dos Profissionais de Enfermagem nas ILPIs visando à segurança e o bem-estar dos residentes dessas instituições. ${ }^{9}$

Uma maneira de prestar uma assistência holística e de qualidade ao idoso institucionalizado é por intermédio da realização da Sistematização da Assistência de Enfermagem (SAE) e implementação do Processo de Enfermagem (PE). No Brasil, tais ações são regulamentadas pela Resolução do COFEN n ${ }^{\circ} 358 / 2009 .{ }^{10}$

O PE é compreendido como o método clínico da profissão, uma prática científica para reconhecimento das condições de saúde/doença que fundamenta a assistência de Enfermagem e auxilia na prevenção, promoção e reabilitação da saúde da pessoa, família e coletividade. ${ }^{11}$ É constituído de cinco etapas: coleta de dados de enfermagem, diagnóstico de enfermagem (DE), planejamento de enfermagem, implementação e avaliação de enfermagem. ${ }^{10}$ Taxonomias de Enfermagem podem/devem ser utilizadas para designar os DEs, resultados e intervenções de Enfermagem.
Entre as taxonomias de Enfermagem, a NANDA International Inc. (NANDA-I) é uma referência universalmente notória e que se tornou um parâmetro respeitado para a identificação de DEs e o define como "julgamento clínico a respeito de uma resposta humana indesejável a uma condição de saúde/processo de vida que existe em uma pessoa, família, grupo ou comunidade". ${ }^{12}$ O DE é capaz de auxiliar na redução de complicações clínicas e diminuição de riscos, além de contribuir com a Enfermagem baseada em evidências científicas. ${ }^{12}$

Ao reconhecer os idosos institucionalizados como um grupo vulnerável e específico, é ressaltada a relevância da Enfermagem na formulação do julgamento clínico e crítico direcionados a prevenção de danos, promoção da saúde e controle das possíveis complicações. ${ }^{13}$ Nesse sentido, diante da importância do tema para a área da saúde e Enfermagem e da vulnerabilidade da população ao problema, percebe-se a necessidade de identificação das demandas de cuidados determinadas pelos DEs, pois tais se apresentam como uma ação relevante para a atuação clínica e científica do enfermeiro.

Diante do exposto, objetiva-se no presente estudo identificar diagnósticos de enfermagem da Taxonomia da NANDA-I em idosos institucionalizados.

\section{MÉTODO}

Trata-se de um estudo descritivo, desenvolvido com idosos de uma ILPI do município de Montes Claros, Minas Gerais, Brasil, no período de junho de 2018 a julho de 2019. Destaca-se que a instituição não utiliza linguagem padronizada de DE e que esse período foi o necessário para se realizar a coleta total de dados dos idosos institucionalizados.

A ILPI atende 120 idosos, 24 horas por dia, tem um quadro de funcionários de formação superior, sendo: Médico, Enfermeiro, Fisioterapeutas, Dentista, Nutricionista, Educador Físico, Assistente Social e Terapeuta Ocupacional. De nível médio: Técnicos de Enfermagem, Cozinheiras, Vigias, Motoristas, Estoquista, Mensageiros, Operadores de Telemarketing, Bombeiro/Eletricista, Serviços Gerais e Administrativos com curso de capacitação de cuidador de idosos.

O universo foi constituído por idosos que atenderam os seguintes critérios de inclusão: idade igual ou superior a 60 anos, estar institucionalizado na ILPI, consentir em participar do estudo ou ter sua participação autorizada pelo responsável/cuidador por meio da assinatura do Termo de Consentimento Livre e Esclarecido (TCLE) ou pelo Termo de Assentimento, respectivamente.

Do total de 120 idosos, participaram do estudo 116, visto que, ocorrem quatro (4) perdas relativas a idosos que se encontravam em internação hospitalar durante o período de coleta de dados na ILPI.

O processo de coleta de dados foi desenvolvido por uma graduanda em enfermagem, do sétimo período do curso de graduação em enfermagem de uma universidade pública do estado de Minas Gerais, qualificada e instruída pelo professor orientador. $\mathrm{O}$ treinamento foi constituído por aprofundamento 
teórico sobre anamnese e o exame clínico, qualificação prática, análises de textos e artigos sobre $o$ tema.

A pesquisa dos dados foi realizada por meio de anamnese e exame clínico dos idosos institucionalizados. A duração média da coleta de dados foi de 1 hora. Foi aplicado um instrumento sistematizado, desenvolvido a partir da literatura ${ }^{14-17}$ constituído por variáveis de caracterização sociodemográfica e clínica. Salienta-se que o instrumento de coleta de dados passou por análise e refinamento de profissionais especializados, com experiência na área de saúde do idoso. Dessa forma, todas as modificações propostas foram realizadas e o instrumento final foi consolidado e estabelecido por consenso.

$\mathrm{O}$ instrumento foi fundamentado no referencial de Wanda Aguiar Horta, tomando-se como modelo teórico norteador a teoria das Necessidades Humanas Básicas (NHBs) ${ }^{16} \mathrm{e}$ constituiu-se pelos seguintes tópicos: identificação e história de saúde (sexo, idade, estado civil, etnia, renda familiar, escolaridade, profissão/ocupação, histórico de saúde, queixa atual, diagnósticos médicos, história medicamentosa, hábitos e estilo de vida), necessidades psicobiológicas (sono/repouso, regulação neurológica, percepção sensorial, cuidado corporal, termorregulação, aparência geral, regulação vascular/circulação, nutrição/hidratação, oxigenação/respiração, exame do abdome, atividade física/mobilidade e integridade tecidual, eliminação, sexualidade), psicossociais (comunicação, interação social, relação familiar, entretenimento, autoestima, autorrealização, segurança, conhecimento sobre doença e tratamento e ambiente) e psicoespirituais (crenças religiosas, necessidades espirituais e manutenção desses hábitos).

Após a realização da consulta de enfermagem, a anamnese $e$ o exame clínico de cada idoso, foram analisados e identificados os termos ou expressões em campos livres das respostas humanas em nível de bem-estar ou que necessitariam de intervenções específicas de enfermagem: condições de saúde, disfunções, condições de vulnerabilidade, processos de vida e motivação para aumentar o bem-estar. Estes foram, assim, a base para identificação dos DEs constantes na taxonomia da NANDA-I, edição 2018/2020. ${ }^{12}$

Para validação dos achados, utilizou-se a técnica de validação por consenso ${ }^{18,19}$ que propõe análise por um grupo particular de enfermeiros clínicos, mínimo de três e máximo de cinco, com intuito de estabelecer opinião consensual (100\%) de especialistas sobre a pertinência e relevância de determinado termo/expressão ou DE. A discordância de um ou mais especialistas condicionou a não validação dos respectivos termos/expressões ou DEs. ${ }^{19}$ Os critérios de inclusão dos especialistas foram: ser enfermeiro(a), ter atuação profissional/residência em saúde, com duração mínima de dois anos de prática clínica de assistência a idosos, autor(a), coautor(a) ou orientador(a) de estudos envolvendo saúde do idoso. Selecionaram-se quatro especialistas, considerando a proximidade geográfica para realização dos encontros presenciais.

$O$ processo de validação por consenso ocorreu em um município do Norte de Minas Gerais, em que quatro especialistas participaram de dois encontros presenciais, e norteados pelas habilidades de sistematização do raciocínio diagnóstico de Risner, ${ }^{20,21}$ validaram os DEs.

Enfatiza-se que, a partir da linha de raciocínio de Risner ${ }^{20,21}$ foi possível aplicar o raciocínio analítico e sintético, considerando as habilidades de pensamento crítico, raciocínio clínico, ${ }^{22} 0$ conhecimento científico e experiências dos enfermeiros em correspondência às inferências elaboradas a partir dos elementos da anamnese e o exame clínico de cada idoso, e a linguagem padronizada adotada na taxonomia da NANDA-I, resultando nos DEs.

Os dados foram adicionados a uma planilha eletrônica Microsoft Excel 2013 e efetuada análise descritiva (frequências simples e percentual).

O presente estudo foi aprovado pelo Comitê de Ética em Pesquisa da Universidade Estadual de Montes Claros (UNIMONTES) sob o número de parecer: 2.536.218 e CAAE: 84391518.9.0000.5146, em 09 de março de 2018.

\section{RESULTADOS}

Entre os 116 idosos, constatou-se que $70(60,3 \%)$ eram indivíduos do sexo masculino. A idade variou entre 60 e 107 anos, com média de 78,2 anos e desvio padrão de 9,21 anos. Foram mais prevalentes os idosos que se declararam pardos $(\mathrm{N}=77$ $66,3 \%$ ), solteiros ( $N=77 ; 66,3 \%$ ), e quanto à profissão/ocupação, houve o predomínio de agricultores ( $\mathrm{N}=38 ; 32,7 \%)$ e do $\operatorname{lar}(\mathrm{N}=27$ $23,2 \%$ ). Quanto ao tempo de institucionalização e escolaridade, $60(51,7 \%)$ idosos estavam institucionalizados entre 1 e 5 anos e $52(44,8 \%)$ possuíam o ensino fundamental I incompleto.

No que se refere às características clínicas, 111 (95,6\%) idosos não apresentavam alergias, $108(93,1 \%)$ não eram etilistas, $92(79,3 \%)$ não eram tabagistas. Quanto às medicações, $49(42,4 \%)$ utilizavam antidepressivos, $46(39,6 \%)$ anti-hipertensivos e $20(17,2 \%)$ drogas vasoativas.

A partir da avaliação foram identificados 2.859 termos que demonstravam alterações nas necessidades humanas básicas dos idosos: situações de vulnerabilidade, disfunções, processos de vida, nas quais a enfermagem deve intervir por meio do PE. Após a eliminação das repetições, foram obtidos 149 termos e manifestações.

A NANDA-I possui 244 DEs classificados em 13 domínios de necessidades humanas ${ }^{(12)}$. Identificou-se 1.555 títulos de DEs para os 116 idosos, com uma média de 13,4 diagnósticos por idoso. Após exclusão de repetições foram obtidos 39 títulos distintos de DEs. Entre eles, 26 (66,7\%) são títulos de DEs reais e $13(33,3 \%)$ são títulos de diagnósticos de risco, e estão classificados em 8 dos 13 domínios da taxonomia da NANDA-I. Destaca-se que não houve identificação de títulos diagnósticos nos domínios 6. Autopercepção; 8. Sexualidade 9. Enfrentamento/Tolerância ao Estresse; 10. Princípios da Vida e 13. Crescimento/Desenvolvimento (Tabela 1).

Os DEs mais frequentes (identificados, no mínimo, em 60\% dos idosos) foram: risco de quedas (00155) ( $\mathrm{N}=110,94,8 \%$; síndrome do idoso frágil (00257) $(\mathrm{N}=106,91,3 \%)$; processos familiares disfuncionais (00063) $(\mathrm{N}=105,90,5 \%)$; memória 
Tabela 1. Distribuição dos títulos diagnósticos de enfermagem identificados em idosos ( $N=116)$ em instituição de longa permanência para idosos, conforme os domínios da NANDA-I. Montes Claros, Minas Gerais, Brasil, 2019.

\begin{tabular}{|c|c|c|}
\hline Domínio & Títulos diagnósticos de enfermagem (código) & n (\%) \\
\hline 1.Proteção da Saúde & Síndrome do idoso frágil (00257) & $106(91,3)$ \\
\hline \multirow[t]{3}{*}{ 2. Nutrição } & Volume de líquidos deficiente (00027) & $12(10,3)$ \\
\hline & Risco de volume de líquidos deficiente (00028) & $97(83,6)$ \\
\hline & Risco de glicemia instável (00179) & $10(8,6)$ \\
\hline \multirow[t]{4}{*}{ 3. Eliminação e Troca } & Constipação (00011) & $16(13,7)$ \\
\hline & Risco de constipação (003015) & $93(80,1)$ \\
\hline & Incontinência urinária de urgência (00019) & $3(2,5)$ \\
\hline & Incontinência urinária funcional (00020) & $62(53,4)$ \\
\hline \multirow[t]{12}{*}{ 4. Atividade/Repouso } & Débito cardíaco diminuído (00029) & $31(26,7)$ \\
\hline & Padrão respiratório ineficaz (00032) & $20(17,2)$ \\
\hline & Deambulação prejudicada (00088) & $32(27,5)$ \\
\hline & Insônia (00095) & $33(28,4)$ \\
\hline & Privação do sono (00096) & $8(6,8)$ \\
\hline & Déficit no autocuidado para alimentação (00102) & $13(11,2)$ \\
\hline & Déficit no autocuidado para banho (00108) & $88(75,8)$ \\
\hline & Déficit no autocuidado para vestir-se (00109) & $79(68,1)$ \\
\hline & Déficit no autocuidado para higiene íntima (00110) & $56(48,2)$ \\
\hline & Risco de perfusão tissular cardíaca diminuída (00200) & $2(1,7)$ \\
\hline & Perfusão tissular periférica ineficaz (00204) & $18(15,5)$ \\
\hline & Risco de pressão arterial instável (00267) & $7(6)$ \\
\hline \multirow[t]{4}{*}{ 5. Percepção/Cognição } & Comunicação verbal prejudicada (00051) & $37(31,8)$ \\
\hline & Confusão aguda (00128) & $23(19,8)$ \\
\hline & Confusão crônica (00129) & $41(35,3)$ \\
\hline & Memória prejudicada (00131) & $101(87)$ \\
\hline 7. Papéis e relacionamento & Processos familiares disfuncionais (00063) & $105(90,5)$ \\
\hline \multirow[t]{11}{*}{ 11. Segurança/Proteção } & Hipotermia (00006) & $4(3,4)$ \\
\hline & Integridade tissular prejudicada (00044) & $17(14,6)$ \\
\hline & Risco de integridade da pele prejudicada (00047) & $34(29,3)$ \\
\hline & Dentição prejudicada (00048) & $90(77,5)$ \\
\hline & Risco de violência direcionada a outros (00138) & $15(12,9)$ \\
\hline & Risco de violência direcionada a si mesmo (00140) & $66(56,8)$ \\
\hline & Risco de quedas (00155) & $110(94,8)$ \\
\hline & Risco de sangramento (00206) & $1(0,8)$ \\
\hline & Risco de ressecamento ocular (00219) & $3(2,5)$ \\
\hline & Risco de integridade tissular prejudicada (00248) & $17(14,6)$ \\
\hline & Risco de lesão por pressão (00249) & $47(40,5)$ \\
\hline \multirow[t]{3}{*}{ 12. Conforto } & Dor aguda (00132) & $1(0,8)$ \\
\hline & Dor crônica (00133) & $56(48,2)$ \\
\hline & Náusea (00134) & $1(0,8)$ \\
\hline
\end{tabular}

Fonte: Pesquisa de Campo, 2019. 
prejudicada (00131) $(\mathrm{N}=101,87 \%)$; risco de volume de líquido deficiente (00028) $(\mathrm{N}=97,83,6 \%)$; risco de constipação (00011) ( $N=93,80,1 \%)$; dentição prejudicada (00048) $(\mathrm{N}=90,77,5 \%)$ déficit no autocuidado para banho (00108) ( $\mathrm{N}=88,75,8 \%$ ) e déficit no autocuidado para vestir-se (00109) $(\mathrm{N}=79,68,1 \%)$.

\section{DISCUSSÃO}

Em ILPI, o enfermeiro exerce função importante na admissão do idoso na instituição, devendo inseri-lo na rotina, apresentar-Ihe a instituição, a estrutura física, os demais residentes e a equipe de profissionais. Deve acolher o idoso de maneira a proporcionar melhor e mais rápida adaptação. Conhecer o perfil dos idosos, as suas fragilidades, o nível de dependência e os Diagnósticos de Enfermagem é fundamental para o planejamento da assistência, para que seja possível implementar um plano de cuidados de maneira individualizada e resolutiva, conforme as necessidades de cada idoso. ${ }^{21,22}$

A partir da análise dos dados, identificou-se 39 títulos diagnósticos. Desses, 26 (66,7\%) referem-se a títulos de DEs reais e $13(33,3 \%)$ a títulos de diagnósticos de risco. Enfatiza-se que os DEs devem ser priorizados conforme o risco iminente à vida. Tais, devem ser identificados e intervenções executadas com a finalidade de evitar complicações e preservar a segurança do paciente. ${ }^{23}$

Os dados de caracterização dos idosos institucionalizados são ratificados em outro estudo que objetivou descrever as características sociodemográficas e clínicas de idosos institucionalizados e constatou uma maior prevalência de idosos do sexo masculino $(61,1 \%)$, solteiros $(46,3 \%)$, analfabetos $(46,3 \%)$ ou de baixa escolaridade $(22,2 \%){ }^{24}$

Enfatiza-se que a baixa escolaridade é muito frequente ao se analisar instituições filantrópicas, visto que resulta da realidade pregressa de discriminação de oportunidades educacionais a esses idosos. No Brasil, reflete-se a associação entre as situações socioeconômicas, a conclusão do ensino fundamental e médio e o acesso ao ensino superior. ${ }^{25}$

Observou-se que, no domínio Segurança/Proteção, o diagnóstico mais frequente foi o Risco de quedas (00155) $(\mathrm{N}=110,94,8 \%)$, definido como a "suscetibilidade aumentada a quedas que pode causar dano físico e comprometer a saúde". ${ }^{12}$ Estudos realizados com idosos institucionalizados em Fortaleza ${ }^{26}$ e na Espanha ${ }^{27}$ corroboram esse resultado.

Entre as caraterísticas intrínsecas, com o processo de envelhecimento, nas alterações musculoesqueléticas, observa-se a diminuição de força muscular ${ }^{28}$ devido relevante redução na dimensão de fibras musculares de contração rápida quando comparadas às fibras de contração lenta, que pode resultar em impactos negativos e progressivos como, alterações na postura do idoso e nas estruturas do aparelho locomotor, propiciando lentidão da marcha e perda do equilíbrio, aspectos esses que conduzem a um maior risco de quedas. ${ }^{29}$

Além dos aspectos intrínsecos, destacam-se também os extrínsecos: pisos escorregadios e/ou desnivelados, ausência de barra de apoio e proteção em escadarias, objetos em ambientes de circulação, degraus muito elevados, iluminação inadequada entre outros que podem expor os idosos a quedas. ${ }^{30,31}$

Nessa perspectiva, o enfermeiro, juntamente com a equipe multiprofissional, deve analisar os possíveis fatores de riscos para quedas, sejam eles intrínsecos ou extrínsecos, e assim planejar e executar ações que possam prevenir e/ou minimizar a ocorrência do problema.

No domínio Promoção da Saúde, foi identificado com maior prevalência, o título diagnóstico Síndrome do idoso frágil (00257) ( $\mathrm{N}=106,91,3 \%$ ), definido como "estado dinâmico de equilíbrio instável que afeta o idoso que passa por deterioração em um ou mais domínios de saúde (físico, funcional, psicológico ou social) e leva ao aumento da suscetibilidade a efeitos de saúde adversos, em particular a incapacidade". ${ }^{12}$ Esse achado é semelhante ao de outro estudo conduzido em ILPI de Ribeirão Preto, São Paulo. ${ }^{32} \mathrm{O}$ termo fragilidade também é frequentemente utilizado para caracterizar o grau de vulnerabilidade do idoso a desfechos adversos, como declínio funcional, quedas, internação hospitalar, institucionalização e óbito. Assim, trata-se de um diagnóstico que analisa os fatores físicos, funcionais, psicológicos e sociais, que possibilita a identificação de vulnerabilidades e fragilidades em idosos. ${ }^{33}$

Constatou-se maior frequência, no domínio Papéis e Relacionamentos, o diagnóstico Processos familiares disfuncionais (00063) ( $\mathrm{N}=105,90,5 \%)$, que segundo a NANDA - I, tem por definição "funcionamento familiar que falha em sustentar o bem-estar de seus membros". ${ }^{12}$ Estudo realizado no Rio de Janeiro confirma essa prevalência. ${ }^{34}$ Diante do exposto, enfatiza-se a importância de incentivar e fortalecer os vínculos e a reinserção do idoso no ambiente familiar, responsabilizando a família pelos seus cuidados. Compreende-se também, que a equipe multiprofissional da instituição de longa permanência possui a função de auxiliar os idosos no processo de institucionalização e ofertar assistência social, emocional, física e mental. ${ }^{5}$

No domínio Percepção/Cognição, observaram-se como mais frequentes os DEs Memória prejudicada (00051) ( $\mathrm{N}=101,87 \%)$ e Confusão crônica (00129) $(\mathrm{N}=41,35,3 \%)$, definidos como "incapacidade persistente de recordar ou recuperar partes de informações ou habilidades" "e "alteração irreversível, progressiva, insidiosa e prolongada do intelecto, do comportamento e da personalidade, manifestada por prejuízo nas funções cognitivas (memória, fala, linguagem, tomada de decisão e função executiva) e dependência na execução das atividades diárias"12 respectivamente.

Estudo realizado com idosos residentes em ILPI de Teresina ${ }^{22}$ apresentou o título diagnóstico Memória prejudicada em 38,9\% dos idosos e Confusão crônica em 25,4\%. Já em estudo do Ceará $^{35}$ a frequência do DE Memória prejudicada foi de $26 \%$. A demência é uma patologia diretamente relacionada ao processo de envelhecimento, determinada por perdas cognitivas que interferem inicialmente na memória, na orientação espaço-temporal, no raciocínio e na capacidade de julgamento. Em fases mais evoluídas, manifesta a perda grave das capacidades cognitivas aproximando-se da dependência total. ${ }^{36}$ Diante dessa conjuntura 
observa-se a necessidade das ILPIs incorporarem, entre as medidas de assistência aos idosos, ações que incentivem a preservação da capacidade cognitiva, e também determinar um cuidado individualizado para os idosos com prejuízo cognitivo. ${ }^{37}$

Identificou-se, no domínio Atividade/Repouso, maior prevalência de DEs, indicando a dependência dos idosos institucionalizados para realização das Atividades Básicas de Vida Diária (ABVD), como banho, vestir-se, higiene íntima e alimentação. Os diagnósticos mais frequentes foram Déficit no autocuidado para banho (00108) $(n=88,75,8 \%)$, definido pela "incapacidade de completar as atividades de limpeza do corpo de forma independente." e Déficit no autocuidado para vestir-se (00109) ( $n=68,1 \%)$, que possui como definição a "incapacidade de vestir e retirar as roupas de forma independente". ${ }^{12}$ Outro estudo realizado no estado da Bahia apresentou achado semelhante. ${ }^{37}$

A funcionalidade é fator essencial na avaliação do processo saúde doença dos idosos, necessitando ser considerada na análise da qualidade de vida dessa população. As Atividades Instrumentais de Vida Diária (AIVD) correspondem às práticas de maior complexidade comparadas às $A B V D$, sendo que essas primeiras indicam maior associação com a convivência social do que com as condições individuais. ${ }^{38}$

Os resultados demonstraram que, devido ao perfil clínico dos idosos institucionalizados, há uma prevalência diversificada de DEs, principalmente relacionados aos domínios da NANDA-I, Atividade/repouso e Segurança/proteção. Porém, não foram elaborados DEs nos domínios de respostas humanas Autopercepção, Sexualidade, Enfrentamento/Tolerância ao estresse, Princípios da vida e Crescimento/Desenvolvimento. Resultado corroborado por outros estudos. ${ }^{13,22}$

O enfermeiro é um profissional essencial nas ILPIs, pois o processo de envelhecimento pode gerar diversas respostas humanas alteradas específicas dessa população, e a identificação dos DEs por meio da aplicação do PE com vistas à implementação da SAE possibilita uma assistência sistematizada, individualizada, e holística impactando na preservação e/ou aumento da funcionalidade dos idosos institucionalizados, melhorando a sua qualidade de vida no processo de envelhecer.

O estudo evidencia algumas limitações, decorrente de um estudo transversal, não tornando possível investigar condições de baixa prevalência, factível em desenhos longitudinais. Salienta-se que devido à carência de literatura nacional e internacional, consequentemente não é possível deduzir que os resultados identificados neste estudo são totalmente correspondentes à realidade clínica.

\section{CONCLUSÃO E IMPLICAÇÕES PARA A PRÁTICA}

O presente estudo possibilitou a identificação de 39 títulos de DEs da NANDA-I em pacientes idosos em ILPI. Atendo-se aos domínios, os diagnósticos mais frequentes foram: risco de quedas, síndrome do idoso frágil, processos familiares disfuncionais e memória prejudicada.
A identificação dos DEs permite a caracterização do perfil da população idosa nesse cenário institucional e proporciona uma avaliação mais ampla do estado de saúde do idoso. A partir dessa identificação, o planejamento e a execução de medidas assistenciais podem ser direcionadas para as reais necessidades dos idosos institucionalizados, otimizando a assistência de enfermagem, além de possíveis impactos na autonomia, independência e aumento da qualidade de vida dos idosos. O perfil de DEs contribui também para o fortalecimento da identidade profissional e da Enfermagem enquanto ciência.

\section{FINANCIAMENTO}

Fundação de Amparo à Pesquisa do Estado de Minas Gerais (FAPEMIG).

\section{CONTRIBUIÇÕES DOS AUTORES}

Desenho do estudo e orientação. Coleta e análise de dados. Interpretação dos resultados. Redação e revisão crítica do manuscrito. Aprovação da versão final do artigo. Responsabilidade por todos os aspectos do conteúdo e a integridade do artigo publicado. Diego Dias de Araújo

Desenho do estudo. Análise de dados e interpretação dos resultados. Redação e revisão crítica do manuscrito. Aprovação da versão final do artigo. Responsabilidade por todos os aspectos do conteúdo e a integridade do artigo publicado. Ricardo Otávio Maia Gusmão

Coleta de dados e interpretação dos resultados. Redação e revisão crítica do manuscrito. Aprovação da versão final do artigo. Responsabilidade por todos os aspectos do conteúdo e a integridade do artigo publicado. Edileuza Teixeira Santana. Gabriella Gonçalves Coutinho. Daniel Vinicius Alves Silva. Tatielle Aparecida Almeira Bernardes. Luiza Rodrigues Camisasca

\section{EDITOR ASSOCIADO}

Candida Caniçali Primo

\section{REFERÊNCIAS}

1. Miranda GMD, Mendes ACG, Silva ALA. Population aging in Brazil: current and future social challengesand consequences. Rev Bras Geriatr Gerontol. 2016;19(3):507-19. http://dx.doi.org/10.1590/180998232016019.150140 .

2. Ministério da Saúde (BR), Secretaria de Atenção à Saúde. Estatuto do idoso [Internet]. Brasília: Ministério da Saúde;2013. 70 p. [citado 23 nov 2019]. Disponível em: http://bvsms.saude.gov.br/bvs/publicacoes/ estatuto_idoso_3edicao.pdf

3. Instituto Brasileiro de Geografia Estatística. Pesquisa Nacional por Amostra de Domicílios Contínua 2012/2017: características gerais dos domicílios e dos moradores 2017 [Internet]. Rio de Janeiro: IBGE; 2018. 8 p. [citado 23 nov 2019]. Disponível em: https://biblioteca.ibge. gov.br/visualizacao/livros/liv101566_informativo.pdf

4. Alencar MA, Bruck NNS, Pereira BC, Câmara TMM, Almeida RS. Perfil dos idosos residentes em uma instituição de longa permanência. Rev Bras Geriatr Gerontol. 2012;15(4):785-96. http://dx.doi.org/10.1590/ S1809-98232012000400017. 
5. Alcântara RKL, Cavalcante MLSN, Fernandes BKC, Lopes VM, Leite SFP, Borges CL. Sociodemographic and health profile of institutionalized elderly people institucionalizados. J Nurs UFPE Online. 2019;13(3):6749. https://doi.org/10.5205/1981-8963-v13i3a237384p674-679-2019.

6. Resolução RDC $n^{\circ} \mathbf{2 8 3}$, de 26 de setembro de 2005 (BR). Regulamento técnico para o funcionamento das instituições de longa permanência para idosos. Diário Oficial da União [periódico na internet], Brasília (DF), 27 set 2005 [citado 23 nov 2019]. Disponível em: http://bvsms. saude.gov.br/bvs/saudelegis/anvisa/2005/res0283_26_09_2005.html

7. Vieira SKSF, Alves EMN, Fernandes MA, Martins MCC, Lago EC. Sociodemographic characteristics and morbidities among institutionalized elderly without cognitive decline. Cuidado é Fundamental. 2017;9(4):11328. http://dx.doi.org/10.9789/2175-5361.rpcfo.v9.5909.

8. Lei $n^{\circ} 7.498$ de 25 de junho de 1986 (BR). Dispõe sobre a regulamentação do exercício da Enfermagem e dá outras providências. Diário Oficial da União [periódico na internet], Brasília (DF), 25 jun 1986. [citado 23 nov 2019]. Disponível em: http://www.cofen.gov.br/lei-n-749886-de25-de-junho-de-1986_4161.html/print/

9. Resolução COFEN no 620/2019, de 4 de novembro de 2019 (BR). Normatiza as atribuições dos Profissionais de Enfermagem nas instituições de Longa Permanência para Idosos - ILPI. Diário Oficial da União [periódico na internet], Brasília (DF), 6 nov 2019. [citado 23 nov 2019]. Disponível em: http://www.cofen.gov.br/resolucao-cofenno-620-2019_74957.html/print/

10. Resolução COFEN no $\mathbf{3 5 8}$, de 15 de outubro de 2009 (BR). Dispõe sobre a Sistematização da Assistência de Enfermagem e a implementação do Processo de Enfermagem em ambientes, públicos ou privados, em que ocorre o cuidado profissional de Enfermagem, e dá outras providências. Diário Oficial da União [periódico na internet], Brasília (DF), 23 out 2009. [citado 23 nov 2019]. Disponível em: http://www. cofen.gov.br/resoluo-cofen-3582009_4384.html

11. Ubaldo I, Matos E, Salum NC, Girondi JBR, Shiroma LB. NANDA International nursing diagnoses in patients admitted to a medical clinic unit. Rev Rene. 2017;18(1):68-75. http://dx.doi.org/10.15253/21756783.2017000100010.

12. Herdman TH, Kamitsuru S. NANDA International nursing diagnoses: Definitions classification, 2018-2020. New York: Theime; 2017. http:// dx.doi.org/10.1055/b-006-161141.

13. Souza LHR, Santana IF, Jesus SS. Capacidade funcional de idosos residentes em uma instituição de longa permanência. Acta Biomed Bras. 2017 dez;8(2):101-10. http://dx.doi.org/10.18571/acbm.144.

14. Garbaccio JL, Ferreira AD. Diagnósticos de enfermagem em uma instituição de longa permanência para idosos. R. Enferm. Cent. O. Min. 2012 set/dez;2(3):303-13. http://dx.doi.org/10.19175/recom.v0i0.218.

15. Freitas MC, Pereira RF, Guedes MVC. Diagnósticos de enfermagem em idosos dependentes residentes em uma instituição de longa permanência em Fortaleza-CE. Cienc Cuid Saúde. 2010;9(3):518-26. http://dx.doi.org/10.4025/cienccuidsaude.v9i3.9544.

16. Horta WA. Processo de enfermagem. Rio de Janeiro: Guanabara Koogan; 2011.

17. Corrêa ESM, Arrabaça MPP, Yamaguchi MU, Bernuci MP. Proposal for an instrument for the admission of the elderly to long-term care facilities: elaboration and validation. Rev Bras Geriatr Gerontol. 2019 nov;22(3):e180215. http://dx.doi.org/10.1590/1981-22562019022.180215.

18. Carlson J. Consensus validation process: a standardized research method to identify and link the relevant NANDA, NIC, and NOC terms for local populations. Int J Nurs Terminol Classif [Internet]. 2006; [citado 23 nov 2019];17(1):23-4. Disponível em: https://www.ncbi.nlm.nih.gov/ pubmed/17117929

19. Félix NDC, Nascimento MNR, Ramos NM, Oliveira CJ, Nóbrega MML. Specialized nursing terminology for the care of people with metabolic syndrome. Esc Anna Nery. 2020;24(3):e20190345. https://doi. org/10.1590/2177-9465-ean-2019-0345.

20. Risner PB. Diagnosis: analysis and synthesis of data. In: Griffith-Kenney JW, Christensen PJ, editores. Nursing process application of theories, frameworks, and models. 2nd ed. St. Louis: Mosby; 1986. p. 124-51
21. Christensen PJ, Kenney JW, editores. Nursing process: application of conceptual models. 4th ed. St. Louis: Mosby; 1995.

22. Carvalho EC, Dalm C, Herdman TH. Contribuição das linguagens padronizadas para a produção do conhecimento, raciocínio clínico e prática clínica da Enfermagem. Rev Bras Enferm. 2013;66:134-41. http://dx.doi.org/10.1590/S0034-71672013000700017.

23. Valcarenghi RV, Santos SSC, Hammerschmidt KSA, Barlem ELD, Gomes GC, Silva BT. Institutional actions based on nursing diagnoses for preventing falls in the elderly. Rev Rene. 2014 mar/abr;15(2):224-32. http://dx.doi.org/10.15253/2175-6783.2014000200006.

24. Bevenides KGCB, Ibiapina A S, de Souza SC, Medino YMS, Ataíde K MN. Clinical framework of elderly people in a long-term institution. J Nurs UFPE Online. 2019 Mar;13(3):594-603. https://doi.org/10.5205/19818963-v13i3a237427p594-603-2019.

25. Araújo DD, Carvalho RLR, Chianca TCM. Nursing diagnoses identified in records of hospitalized elderly. Invest Educ Enferm. 2014;32(2):22535. http://dx.doi.org/10.17533/udea.iee.v32n2a05. PMid:25230033.

26. Borges CL, Silva MJ, Clares JWB, Bezerra JWB, Nogueira JM, de Freitas MC. Sociodemographic and clinical characteristics of institutionalized older adults: contributions to nursing care. Rev Enferm UERJ. 2015 jul;23(3):381-7. http://dx.doi.org/10.12957/reuerj.2015.4214.

27. Cordeiro LM, Paulino JL, Bessa MEP, Borges CL, Leite SFP. Quality of life of frail and institutionalized elderly. Acta Paul Enferm. 2015;28(4):361-6. http://dx.doi.org/10.1590/1982-0194201500061.

28. Pinheiro NCG, Holanda VCD, Melo LA, Medeiros AKB, Lima KC Inequality in the characteristics of the institutionalized elderly in the city of Natal, Brazil. Cien Saude Colet. 2016 nov;21(11):3399-405. http:// dx.doi.org/10.1590/1413-812320152111.19472015. PMid:27828573.

29. Fernandes BKC, Clares JWB, Borges CL, Nóbrega MML, Freitas MC. Nursing diagnoses for institutionalized elderly people based on Henderson's theory. Rev Esc Enferm USP. 2019 jun;53:e03472. http:// dx.doi.org/10.1590/s1980-220x2018004103472. PMid:31166459.

30. García BP, González SM, Muñoz AMC, Antón-Solanas I, Caballero VG, Vela RJ. Risk of drug-related falls among noninstitutionalized older adults. Rev Esc Enferm USP. 2018;52:e03319. http://dx.doi.org/10.1590/ S1980-220X2017012603319. PMid:29668792.

31. Tansini S, Kummer JA, Borges AM, Vidmar MF, Dal Molin V, Wibelinger LM. Influência do exercício resistido na força muscular de idosos. Lect Educ Fis Deporte [Internet]. 2014; [citado 23 nov 2019];19(192). Disponível em: https://www.efdeportes.com/efd192/exercicio-resistidona-forca-muscular-de-idosos.htm

32. Smith AA, Silva AO, Rodrigues RAP, Moreira MASP, Nogueira JA, Tura LFR. Assessment of risk of falls in elderly living at home. Rev Lat Am Enferm. 2017;25(e2754):1-9. http://dx.doi.org/10.1590/15188345.0671.2754. PMid:28403333.

33. Chehuen JA No, Brum IV, Braga NAC, Gomes GF, Tavares PL, Silva RTC et al. Fall awareness as a determining factor of this event among elderly community residents. Geriatr Gerontol Aging. 2017;11(1):25-31. http://dx.doi.org.10.5327/Z2447-211520171600074.

34. Silva TG, Souza PA, Santana RF. Adequacy of nursing language to the practice with elderly residents in a long term psychiatric institution: cross mapping. Cuidado é Fundamental. 2015;7(4):3467-78. http:// dx.doi.org/10.9789/2175-5361.2015.v7i4.3467-3478.

35. Fluetti MT, Fhon JRS, Oliveira AP, Chiquito LMO, Marques S. The frailty syndrome in institutionalized elderly persons. Rev Bras Geriatr Gerontol. 2018;21(1):62-71. http://dx.doi.org/10.1590/1981-22562018021.170098.

36. Esquenazi D, Silva SRB, Guimarães MAM. Aspectos fisiopatológicos do envelhecimento humano e quedas em idosos. Revista HUPE. 2014;13(2):11-20. http://dx.doi.org/10.12957/rhupe.2014.10124.

37. Oliveira PB, Tavares DMS. Condições de saúde de idosos residentes na Instituição de Longa Permanência segundas necessidades humanas básicas. Rev Bras Enferm. 2014;67(2):241-6. PMid:24861067.

38. Nascimento HG, Figueiredo AEB. Dementia, family caregivers and health service: the care of yourself and the other. Cien Saude Colet 2019;24(4):1381-92. http://dx.doi.org/10.1590/1413-81232018244.01212019. PMid:31066840. 